\title{
A PESSOA COM HIPERTENSÃO ARTERIAL EM TRATAMENTO NO AMBULATÓRIO: CONSIDERAÇÕES GERAIS* \\ Parte I
}

Angela Maria Geraldo Pierin**

PIERIN, A.M.G. A pessoa com hipertensăo arterial em tratamento no ambulatório: consideraçoes gerais. Parte 1. Rev. Esc. Enf. USP, São Paulo, 22(2):223-229, ago. 1988.

O presente estudo analisa a problematica que envolve a pessoa com hipertensdo arterial em tratamento ambulatorial e a importância da assisténcia de enfermagem.

UNITERMOS: Hipertensdo. Atendimento ambulatorial.

\section{INTRODUÇĀO}

A pessoa portadora de hipertensão arterial, submetida a tratamento ambulatorial, pode apresentar certos problemas que eventualmente dificultam o controle satisfatório dos niveis tensionais. Ao caracterizar os pŗincipais problemas que ocorrem na vida destas pessoas, a enfermeira, embasada em dados reais e concretos, terá subsídios para planejar e prestar assistência de enfermagem individualizada, que poderá auxiliar o controle dos niveis tensionais.

$\mathrm{O}$ controle dos hipertensos tem sido objeto de questionamentos e estudos, exigindo da equipe de saúde especial atenção e uma programação conjunta, visando a manutenção dos niveis pressóricos dentro de limites considerados ideais. Contudo, a prática tem demonstrado que a efetivação do tratamento não é tarefa fácil, apesar da eficiência das medidas terapêuticas atualmente existentes.

Alguns pacientes, principalmente os assintomáticos, recusam-se aceitar o tratamento. É comum ouvir dos hipertensos questionamentos tais como: "Nåo sinto problemas, por que devo tomar remédios?" ou então "Não preciso continuar a tomar remédios, pois a pressão já está baixa" ou mesmo "Vou deixar de tomar os remédios pois estão fazendo mal."

DANIELS \& KOCHAR (1979) salientaram que a taxa de não aderência ao tratamento anti-hipertensivo está por volta de 30\%. PODELL (1979) apontou que os indices de recusa a tomar os medicamentos variam bastante, porém, informam que um terço dos pacientes sempre toma seus remédios, outro terço toma-os às vezes e, finalmente, um terço raramente ou nunca os toma.

- Extraido da monografia de Mestrado apresentada à EEUSP.

- Enfermeira. Mestre em Enfermagem. Professora Assistente do Departamento de Enfermagem Médico-Cirúrgica da EEUSP - disciplina Enfermagem Médico-Cirürgica. 
Condutas especificas de enfermagem têm sido adotadas com a finalidade de se obter maior adesão dos hipertensos ao tratamento. Uma dessas condutas é a orientação sistematizada de enfermagem, com abordagem dos aspectos relacionados à terapêutica medicamentosa, como dose, horário, indicação, efeitos, dieta e sua composição, quantidade de calorias e de sal e hábitos de vida tais como o uso de fumo e de bebida alcóolica, bem como atividades físicas, dentre outros.

A enfermeira, ao exercer o papel de educadora, promove condiçðes favoráveis, junto ao cliente, para o esclarecimento de dúvidas, o fortalecimento de hábitos de saúde e o direcionamento para o auto cuidado.

Cabe salientar que outros elementos necessitam ser considerados na educação dos hipertensos. Os programas estabelecidos devem atender às reais necessidades da população. A enfermeira, ao implementar programas de educação para a saúde, individuais ou para grupos, precisa avaliar determinados aspectos da questão, tais como: situação social da clientela, nível educacional, idade, atividades que executa, sentimentos e conhecimentos sobre a doença, crenças de saúde, estilo de vida, dentre outros (COHEN, 1981; SYRED, 1981; DANIELS, 1979). Nesta mesma linha FOSTER (1981) acrescenta outros dados que estão diretamente ligados à aderência do hipertenso ao tratamento, expressos a seguir: experiência anterior com a hipertensão arterial no meio em que vive, percepção da seriedade do problema, complexidade do tratamento, atitudes decorrentes do sistema de saúde vigente, efeitos colaterais da medicação e percepção social do problema. SILVA et alii (1984) salientam que motivos econômicos, instituição de esquemas terapêuticos complicados, aparecimento de efeitos colaterais e relacionamento inadequado entre médico e paciente merecem destaque na adesão do hipertenso ao tratamento.

A consulta de enfermagem foi relatada por FERREIRA \& BRANT(1984) como uma forma de abordar o hipertenso. As autoras observaram que: "É necessaŕio levar o individuo a participar constantemente do tratamento, o sucesso do trabalho dependerá das relaçðes interpessoais enfermeiro-cliente; e este deve ouvir com atenção as queixas do cliente para dar-lhe as informaçðes necessárias. O enfermeiro, no processo educativo, deve informar sobre a morbidade e os riscos da falta de controle; porém é o próprio cliente quem deve identificar e decidir pela importância deste controle em sua situação específica".

Acredita-se que a enfermeira deve atuar de forma participante junto ao hipertenso, utilizando o papel de educadora como um modo de atuação que possibilite um tipo de relacionamento de apoio, visando cada vez mais a participação ativa do cliente em seu auto cuidado: somente a partir do momento que ele estiver envolvido o suficiente com toda a problemática do "continuum" saúde-doença é que se poderá controlar satisfatóriamente seus niveis de pressão arterial.

CHIAVERINI (1980) apontou que $90 \%$ dos hipertensos sofrem de hipertensão arterial essencial ou primária, cuja causa básica é desconhecida; este fato leva à crença de que a doença seja resultante da alteração de vários fatores e que os níveis tensionais elevados são, na maioria das vezes, passiveis de controle mas não de cura.

SILVA et alii (1984), ao esquematizarem a gênese da hipertensão arterial essencial, enfatizam a participação de fatores, tais como a hereditariedade e o ambiente, que, atuando sobre o organismo humano, levam-no a distúrbios na regulação da pressão arterial; entre os fatores ambientais destaca-se a ingestão de sal; estudos epidemiológicos e experimentais revelaram estreitas relaçðes entre a hipertensão arterial e a quantidade de sal na dieta. Em populaçðes indígenas da Amazônia, onde se ingerem quantidades minimas de sal, a hipertensão arterial não foi detectada; por 
outro lado, no Japão, onde o consumo deste ingrediente atinge quantidades elevadas, a hipertensão arterial acomete cerca de $40 \%$ da população. Estes mesmos autores concluíram que pessoas, com predisposição genética, desenvolvem a hipertensão arterial quando ingerem dieta rica em sal, enquanto outros, sem o fator genético, seriam a ela resistentes.

Segundo PAGE (1976), BRESELIN \& SWINTON (1980), LUNA (1980), e SIL$V A$ et alii (1984), a hipertensão arterial afeta de 10 a $20 \%$ a população adulta nos paises industrializados.

Estudos feitos no Brasil, por RIBEIRO et alii (1982), mostraram que na Grande Sảo Paulo a incidência de individuos hipertensos era de $18,1 \%$ nos homens e $6,6 \%$ nas mulheres. No Rio Grande do Sul, estudo relatado por LUNA (1980) revelou essa incidência em $11 \%$ na populaçåo adulta.

Com base nestes dados, considera-se importante proporcionar ao hipertenso, condiçðes que favoreçam controle eficaz da sua pressåo arterial. Ao atuar junto a estas pessoas, constata-se que muitas vezes elas recorrem ao tratamento médico, buscando solução para a cura da pressão elevada. LUNA (1980) afirmou que cerca de $60 \%$ da população hipertensa é identificada e apenas um terço $(20 \%)$ está sendo tratada adequadamente. Por considerar-se grande o contingente de hipertensos não controlados, quer por desconhecimento da doença ou por inadequação do tratamento, supõe-se que, ao realizar um trabalho contando com a participaçăo de vários elementos da equipe multiprofissional, possivelmente obter-se-ao melhores resultados na deteç̧ão e controle das pessoas hipertensas.

A hipertensão arterial, quando não tratada adequadamente, traz consequêências graves para órgãos vitais como cérebro, coração e rins que, uma vez comprometidos podem trazer seqüelas irreversiveis ou levarem o individuo à morte. O estudo de Framinghan revelou que a hipertensão arterial é o principal fator precursor de insuficiência cardiaca e que os hipertensos apresentam de duas a quatro vezes mais infarto do miocárdio do que as pessoas com pressão arterial normal e são quatro vezes mais propensos a acidente vascular cerebral bem como a hipertensão arterial afeta a função renal (CHIAVERINI et alii, 1980). Outros estudos reafirmam estes dados, tais como o de THONSON (1978); este trabalhou com pacientes portadores de acidente vascular cerebral e identificou ser a hipertensão arterial o distúrbio associado que apareceu com maior freqüência (31\%). BARRETO (1980) apontou o acidente vascular cerebral, a insuficiência coronariana, a insuficiência renal e os fenômenos arterioscleróticos como conseqüências de hipertansão arterial severa.

Em face destes dados, considera-se imprescindivel que o hipertenso siga o tratamento instituído, pois este reverterá em benefícios, os quais garantem sobrevida prolongada, destituida de alteraçōes indesejáveis (BRESLIN \& SWINTON, 1980; HUTCHINS, 1981).

Vários autores são unânimes ao afirmarem que a educação para a saúde dos individuos hipertensos é ponto relevante para o êxito no controle da pressão arterial elevada. Reforçam, também, a necessidade do cliente aprender o auto cuidado; aqui a enfermeira deve atuar amplamente como elemento central diretivo do processo de educação (LINDE, 1979; GRANCIO, 1981; LOWTHER, 1981; SYRED, 1981; FINK, 1981; SPRATLEN, 1982).

NAKAMAE (1979), ao falar sobre a importância do papel desempenhado pela enfermeira na terapêutica medicamentosa anti-hipertensiva, destaca que a sua atuação vai desde o conhecimento do paciente quanto à droga a ser utilizada, o ajustamento da dose, o controle dos efeitos desejáveis, até a motivação do paciente para 
participar ativamente no próprio tratamento; ressaltou a importância da orientação do paciente no que se refere à ingestão de drogas anti-hipertensivas, a fim de ser obtida ampla colaboração deste no seu tratamento. Quanto à interrupção do tratamento, a autora referiu, como possiveis causas, falta de informaçoes adequadas ao paciente sobre o assunto, efeitos colaterais dos medicamentos, falta de apoio familiar, alto custo das drogas, tratamento longo, recusa do paciente em considerar-se doente, e baixo nivel cultural que dificulta a compreensåo do problema.

WATSON (1979) ao realizar estudo com grupo de pessoas hipertensas, submetidas a um programa de orientação, do qual faziam parte educadores de sáude, nutricionistas, enfermeiras e médicos, verificou participação mais significativa e maior interesse daqueles que faziam parte do programa educativo. ROSENFELD \& SILVERBERG (1979) relataram que a atuação de enfermeiras, juntamente com a equipe médica, na educaçăo de hipertensos, elevou a taxa de controle de $39,2 \%$ para $72,8 \%$, e a de abandono foi reduzida de $30 \%$ para $0,65 \%$. WEBB (1980), ao estudar a efetividade de programas de educação na aderência ou não de hipertensos ao tratamento, ressaltou a importância da atividade de enfermeira como educadora.

SECAF (1977) destacou que, dentre os profissionais de saúde, a enfermeira é quem tem maior número de oportunidades para desenvolver atividades junto ao paciente. POHL (1971) estabeleceu que "A tarefa de ensinar é uma das atividades que a enfermeira desempenha a fim de concretizar o objetivo principal da enfermagem: a promoção da saúde".

Segundo SILVEIRA (1976), KAMIYAMA (1977) e CARNEIRO (1983) a enfermeira tem atuação relevante no processo de educaçăo da população e, ao utilizar seus conhecimentos cientificos, presta informaçðes adequadas que são fundamentais para uma aproximação do nivel ideal de saúde.

Estudo comparativo, com o objetivo de avaliar o efeito da orientação no controle dos hipertensos, evidenciou que um grupo experimental, submetido à orientaçăo, apresentou escores mais elevados, em testes de conhecimentos específicos sobre a hipertensão, do que o grupo de controle (TANNER \& POOSER, 1981).

Estudos realizados por POWERS \& WOOLDRIDGE (1982), com a finalidade de identificarem fatores que influenciam atitudes, conhecimentos e aderência ao tratamento de hipertensos, revelaram que programas de educação podem aumentar conhecimentos e responsabilidade do paciente para com o auto-cuidado. Estes estudos, porém, não descobriram correlaçăo direta entre a educaçăo do hipertenso e a diminuição da pressão arterial, demonstrando que somente o aumento de conhecimento não promoveria elevação no indice de aderência ao tratamento instituido; concluiu-se, portanto, que outros fatores intrinsecos à condição do próprio indivíduo dificultam essa adesão. HENSON (1981) apontou possiveis problemas que dificultam o tratamento da pessoa com pressão alta, tais como: medo, recusa em aceitar um tratamento longo, falta de apoio familiar, cronicidade da doença, custo do tratamento, crenças sócio-culturais, estilo de vida, efeitos dos medicamentos e não compreensão das instruçðes recebidas.

CARRASCO et alii (1979), ao identificarem as causas de abandono do tratamento na hipertensão arterial, verificaram que a impossibilidade financeira para comprar remédios, a impressão de já estar curado e os efeitos colaterais dos medicamentos contribuiram com $30 \%, 13 \%$ e $10 \%$, respectivamente, para o absenteísmo.

A dificuldade financeira para comprar os medicamentos, é um fator que merece destaque especial dentro da problemática do controle do hipertenso. Atualmente vive-se um periodo de instabilidade econômica, o que sem dúvida atinge diretamente o 
custo do tratamento, principalmente para pessoas pertencentes às classes sócio-económicas menos favorecidas. ROCCA (1984), ao analisar a economia brasileira, identificou que a inflaçăo em 1984, em ascensăo acelerada, quando comparada à dos anos de 1982 e 83, constituiu um obstáculo ao crescimento econỏmico, gerando desta forma intranquilidade social. A pesquisadora, que atua junto a uma clientela hipertensa, parte deste contexto social e econômico, verificou freqüentemente que muitos deles nåo estavam fazendo o tratamento medicamentoso devido à falta de recursos financeiros para a aquisiçào de remédios.

Quando a autora propós-se realizar o presente estudo, considerou que ao estabelecer um plano assistencial para a pessoa hipertensa, a enfermeira deve ter conhecimento das caracteristicas da populaçăo, inclusive daquelas ligadas ao meio ambiente. CAMPOS (1984) comentou que os niveis de pressào arterial são influenciados por situaçðes estressantes, onde eståo intimamente ligados mecanismos psíquicos e fisicos; os psiquicos resultam de experiências de vida, às quais se somariam outros fatores como idade, estado de saúde, educaçăo, crenças, suportes sociais, intensidade e tipo de experiências atuais, dentre outros.

Ao estabelecer os objetivos da assistência de enfermagem aos hipertensos, a enfermeira deve levar em conta essas caracteristicas para que possa identificar os facilitadores e os dificultadores da consecuçăo dos objetivos traçados inicialmente, visan. do a manutençăo do controle da pressão arterial, sem contudo modificar substancialmente a vida destas pessoas.

Revisando a literatura pertinente, verificou-se a inexistência, em nosso meio, de trabalhos que caracterizam os problemas, as dificuldades, as expectativas destes clientes quanto à sua doença e ao respectivo tratamento. Analisando-se a assistência de enfermagem prestada aos hipertensos, em ambulatórios, observou-se, na maioria das vezes, a existência de açós especificas e padronizadas que conduzem uma programação baseada em orientaçðes individuais ou em grupos.

A prática do dia a dia tem revelado a necessidade de um contato da enfermeira com o cliente, que possibilite condiçסes para que ele verbalize seus problemas. PENDER (1974) salientou que ao ajudar os pacientes a verbalizarem suas necessidades cognitivas referentes à sua condição e seu tratamento, a enfermeira estará identificando as informaçoes que deverăo ser acrescentadas ao plano assistencial.

A enfermeira pode ajudar o hipertenso a identificar condiçðes que contribuem para a elevação do seu nivel tensional e estabelecer com ele um plano assistencial que favoreça o controle de sua doença. Segundo BELAND \& PASSOS (1979), a enferdar ao paciente apoio emocional e ao mesmo tempo orientaçăo quanto à doença e ao tratamento.

Considera-se, também, de real importância, que a enfermeira, ao levantar dados sobre a pessoa com hipertensão arterial, the ofereça condiçoes que facilitem a verbalização dos mesmos. A atuação da autora junto a estas pessoas possibilitou verificar que muitas vezes elas procuram, na enfermeira, alguém em quem confiar, que escute seus problemas de ordem física, social, familiar, econômica e emocional; a maioria das vezes desejam nåo só esclarecimentos para as suas dúvidas, como, também, alguém que compreenda seus anseios. O papel da enfermeira como educadora, dirigindo o hipertenso para o auto cuidado e usando a orientaçăo como forma de abordagem, deverá contribuir para aumentar a aderência do hipertenso ao tratamento. Porém, faz-se necessário que, para tanto, ela tenha subsidios que norteiem suas açōes em todos os pontos da programação estabelecida, o que poderá ser conseguido por meio de conhecimento das reais caracteristicas da clientela. 
PIERIN, A.M.G. The person with the arterial hypertension in ambulatory treatment: general considerations. Part I. Rev. Esc. Enf. USP, Så Paulo, 22(2):223-229, Aug. 1988.

The present study analyses the problems related to the person affected by arterial hypertension, under ambulatory treatment and the importance of nursing care.

UNITERMS: Hypertension. Ambulatory care.

\section{REFERENCIAS BIBLIOGRÁFICAS}

1. BARRETO, A.C.P. Complicaçðes cardiovasculares da hipertensão arterial. IN: SIMPÓSIO INTERNACIONAL. SOBRE HIPERTENSÃO ARTERIAL E SUAS COMPLICAÇŌES, São Paulo, ago. 1980. p. 43-48.

2. BELAND, I. \& PASSOS, J. Enfermagem clínica. Săo Paulo, EPU, 1979. v.2, p. 173-184.

3. BRESLIN, 1. \& SWINTON, W.N. Hypertension and cerebrovascular disease. Primary Care, Philadelphia, 7(1):49-59, Mar. 1980.

4. CAMPOS, E.P. Fisiopatologia da hipertensåo arterial, uma perspectiva integrada. Dílogego Méd., Sào Paulo, 10(4):30-34, 1984.

5. CARNEIRO, T.G. et alii. A importância da educaçăo em sáude desenvolvida nas salas de espera do ambulatório do Hospital Universitário Regional do Norte do Paraná. Trabalho apresentado no CONGRESSO BRASILEIRO DE ENFERMAGEM, 35, Sגo Paulo, 24-30. set. 1983.

6. CARRASCO, R.M. et alii. Causas de abandono do tratamento na hipertensato arterial. Ars. Curandi Cardiol., Rio de Janeiro, 1(8):54-61, fev. 1979.

7. CHIAVERINI, R. et alii. Doença hipertensiva: diagnóstico, etiopatogénese, tratamento. Rio de Janeiro, Atheneu, 1980. $311 \mathrm{p}$.

8. COHEN, S.A. Patient education: a review of the literature. J. Adv. Nurs., Oxford, 6(1): 11-18, Jan. 1981.

9. DANIELS, L.M. \& KOCHAR, M.S. What influences adherence to hypertension therapy. Nurs. Forum, Hillsdale, 18(3):231-245, Aug. 1979.

10. FERREIRA, A.A. \& BRANDT, M.J.C.G.C. Aluaçăo do enfermeiro no auto cuidado do cliente hipertenso. Trabalho apresentado no CONGRESSO BRASILEIRO DE ENFERMAGEM, 36, Belo Horizonte, 28 jul.-ago. 1984.

11. FINK J.W. The challenge of high blood pressure control. Nurs. Clin. North Am., Philadelphia, 16(2):301-8, June 1981.

12. FOSTER, S.B. \& KOUSCH, D. Adherence to therapy in hypertensive patients. Nurs. Clin. North Am., Philadelphia, 16(2):331-41, June 1981.

13. GRANCIO, S.D. Opportunities for nurses in high blood pressure control. Nurs. Clin. North Am., Philadelphia, 16(2):309-20, June 1981.

14. HENSON, M.A. Long term control of hypertension. Nurs. Clin. North Am., Philadelphia, 16(2):343-7, June 1981.

15. HUTCHINS, L.N. Drug treatment of high blood pressure. Nurs. Clin. North Am., Philadelphia, 16(2):365-376, June 1981.

16. KAMIYAMA, Y. et alii. Educaçào para a saúde: experiência de integraçào de hospital-escola de enfermagem. Enf. Novas Dimens., São Paulo, 3(5):300-305, set./out. 1977.

17. LINDE, B.J. \& JANS, N.M. Effect of a teaching program on knowledge and compliance of cardiac patients. Nurs. Res., New York, 28(5):282-286, Sept./Oct. 1979.

18. LOWTHER, N. \& CARTER, V.D. How to increase compliance in hypertensives. Am. J. Nurs., New York, 81(5):963, May 1981.

19. LUNA, R.L. Epidemiologia da hipertensào arterial. In: Simpósio INTERNACIONAL SOBRE HIPERTENSÃo ARTERIAL E SUAS COMPLICAÇÕES, Săo Paulo, ago. 1980. p. 21-33.

20. NAKAMAE, D.D. Anti hipertensivos em enfermagem em geral. Rev. Esc. Enf. USP, Såo Paulo, 11(1):20-27, abr. 1977.

21. PAGE, B.L. Epidemiologic evidence on the etiology of human hypertension and its possible prevention. Am. Heart. J., Saint Louis, 91(4):\$27.534. Apr. 1976.

22. PENDER, N.J. Patients identification of health information received during hospitalization. Nurs. Res., New York, 23(3):262-267, May/June 1974.

23. PODELL, R.N. Manual do medico sobre controle do paciente na hipertension. Såo Paulo, Merk Sharp Dohme do Brasil, 1976. 35 p. 
24. POHL, M.L. Teaching activities of the nursing practioner. Nurs. Res., New York, 14(1):4-11, Jan. 1971.

25. POWERS, M. \& WOOL.DRIDGE, P. Factors influencing knowledge, attitudes and compliance of hypertensive patients. Res. Nurs. Health, New York, 5(4):171-182, Dec. 1982.

26. RIBEIRO, M.D.R. el alii. Prevalência da hipertensão arterial na força de trabalho da Grande Săo Paulo: influência da idade, sexo c grupo étnico. Rev. Assoc. Med. Bras., Săo Paulo, 28(9/10):209-211, se1./our. 1982.

27. ROCCA, C.A. Economia: as barreiras no caminho da recuperação. Prob. Bras., Såo Paulo, 21(241):4-20, now. 1984.

28. ROSENIEL.D, J.B. \& SILVERBERG, D.S. Status of hypertension treatment in Israel. Isr. J. Med. Sci., Jerusalèm, 15(12):1014-1016, Dec. 1979.

29. SECAF, V. Atividade educativa da enfermeira: preparo e desempenho. Sảo Paulo, 1977. $110 \mathrm{p}$. (Dissertaçào de mestrado - Escola de Enfermagen da USP).

30. SILVA, H.B. et alii. Hipertensăo arterial. In: MARCONDES, M. et alii. Clínica médica propedêutica e fisiologia. Rio de Janeiro, Guanabara Koogan, 1984. cap. 21, p. 838-862.

31. SILVEIRA, C.C.X. A importância das informaçđes ao paciente recém hospitalizado. Salvador, 1976. 125 p. (Tese de livre docência - Escola de Enfermagem da Universidade Federal da Bahia).

32. SPRATLEN, L.P. Nurse role dimensions of a school based hypertension screening, education and follow up program. J. Sch. Health, Kent, 52(3):174-178, Mar. 1982.

33. SYRED, M.E.J. The abdications of the role of health education by hospital nurses. J. Adv. Nurs., Oxford, 6(1):27-33, Jan. 1981.

34. IANNER, G.A. \& POOSER, D.J. The effect of instruction on control of blood pressure in individuals with essencial hypertension. J. Adv. Nurs., Oxford, 6(1):99-106, Jan. 1981.

35. THONSON, N.C. Incidence and prognosis of acute cerebrovascular accident admissions to a teaching hospital during one year. Br. J. Clin. Pract., London, 32(71):189-190, July 1978.

36. WATSON, D.S. Health education for hypertensive patients. Aust. Fam. Physician, Sydney, 8:315-320, Mar. 1979.

37. WEBB, P.A. Effectiveness of patient education and pysichosocial conseling in promotion compliance and control among hypertension patients. J. Fam. Pract., San Francisco, 1016): 1047-1055, 1980. 